\title{
Prevalência de enteroparasitoses em crianças de zero a doze anos no Brasil: Revisão integrativa
}

\section{Prevalence of enteroparasitosis in children from zero to twelve years in Brazil: An integrative review}

DOI: $10.46919 / \operatorname{archv3n1-004~}$

Recebimento dos originais: 31/01/2021

Aceitação para publicação: 28/02/2022

\section{Fernanda Klein Gomes}

Acadêmica de Medicina da Faculdade de Ciências Médicas de São José dos Campos - FCMSJC Humanitas.

Faculdade de Ciências Médicas de São José dos Campos - FCMSJC - Humanitas. Avenida Cambui, 5900 - Vila Tatetuba, São José dos Campos - São Paulo - CEP: 12220-611 E-mail: fernandakleingomes@gmail.com

\author{
Antonio Sérgio Mathias \\ Acadêmico de Medicina da Faculdade de Ciências Médicas de São José dos Campos - FCMSJC- \\ Humanitas. \\ Faculdade de Ciências Médicas de São José dos Campos - FCMSJC - Humanitas. \\ Avenida Cambui, 5900 - Vila Tatetuba, São José dos Campos - São Paulo - CEP: 12220-611 \\ E-mail: antoniosergio.mathias@gmail.com
}

\section{Sonia Maria Cursino dos Santos}

Bióloga e Docente do Curso de Medicina da Faculdade de Ciências Médicas de São José dos Campos FCMSJC-Humanitas.

Faculdade de Ciências Médicas de São José dos Campos - FCMSJC - Humanitas. Avenida Cambui, 5900 - Vila Tatetuba, São José dos Campos - São Paulo - CEP: 12220-611

E-mail: sonia.santos@humanitas.edu.br

\section{Alessandra Lorenti Ribeiro}

Médica ginecologista e Docente do Curso de Medicina da Faculdade de Ciências Médicas de São José dos Campos - FCMSJC-Humanitas.

Faculdade de Ciências Médicas de São José dos Campos - FCMSJC - Humanitas. Avenida Cambui, 5900 - Vila Tatetuba, São José dos Campos - São Paulo - CEP: 12220-611

E-mail: alessandra.ribeiro@ humanitas.edu.br

\section{RESUMO}

As doenças enteroparasitárias simbolizam um problema de saúde pública no Brasil. Diversos estudos indicam que as crianças são as mais afetadas principalmente devido a questões de higiene e imunes. Objetivo: Analisar os dados das principais pesquisas existentes no Brasil sobre enteroparasitoses entre préescolares relacionando com aspectos sociais, econômicos e culturais. Método: Foi utilizado uma revisão integrativa para analisar os principais artigos publicados entre 1990 e 2020 sobre a prevalência de parasitoses intestinais em crianças. Resultado: A grande maioria dos estudos foram publicados após 2011 e não houve diferença significativa da prevalência entre as décadas. O método Hoffman, Pons e Janer foi o método mais utilizado (56\%). Parasitas comensais estavam presentes em 21 artigos, refletindo a precariedade ou falta do saneamento básico da cidade dos estudos. Conclusão: A melhor alternativa para a 
redução desta prevalência seria optar pela educação sanitária desde a infância e a orientar os pais uma vez que baixos níveis de escolaridade estão relacionados a alta prevalência de parasitoses.

Palavras-chave: Doenças Parasitárias, Pré-escolares, Criança, Prevalência.

\begin{abstract}
Enteroparasitic diseases symbolize a public health problem in Brazil. Several studies indicate that children are the most affected ones mainly due to lack of hygiene and immune system issues. Objective: To analyze data from the main researches in Brazil on intestinal parasitosis among preschool children, relating to social, economic and cultural aspects. Method: This integrative review analyzed the main articles published between 1990 and 2020 on the prevalence of intestinal parasites in children. Result: The majority of studies were published after 2011 and there was no significant difference in prevalence between decades. The Hoffman, Pons and Janer method was the most used method (56\%). Commensal parasites were present in 21 articles, reflecting the precariousness or lack of basic sanitation in the studied city. Conclusion: The best alternative to reduce this prevalence would be to opt for health education from childhood and to guide parents, since low levels of education are related to the high prevalence of parasitosis.
\end{abstract}

Keywords: Parasitic Diseases, Preschoolers, Child, Prevalence.

\title{
1 INTRODUÇÃO
}

É evidente que as parasitoses intestinais são um problema de saúde pública visto que as más condições de saneamento básico acarretam no aparecimento dessas doenças ${ }^{1}$. Segundo o Sistema Nacional de Informações sobre Saneamento (SNIS), em 2018, quase 100 milhões de brasileiros não têm acesso à coleta de esgoto e 35 milhões de brasileiros não têm acesso a água tratada². Sabe-se que saneamento básico precário, água não tratada e alimentos contaminados são as formas de transmissão mais comuns de enteroparasitoses ${ }^{3}$.

A faixa etária mais acometida pelas parasitoses intestinais é a infantil, prevalente entre 0-12 anos. Isso se deve ao fato das crianças não saberem se higienizar corretamente quando sozinhas e devido ao seu sistema imunológico estar em desenvolvimento. Outro fator para a alta prevalência em crianças é em virtude da inserção das mulheres no mercado de trabalho ${ }^{4}$. Isso proporcionou a sua saída do ambiente domiciliar sendo uma alternativa para o cuidado dos filhos colocarem em creches em período integral, deixando-os suscetíveis às infecções devido ao convívio contínuo entre as crianças ${ }^{4,5}$. Em contra partida, a atenção à saúde da criança tem sofrido diversas mudanças com a incorporação de novas tecnologias para a melhoria de qualidade de vida para essa faixa etária ${ }^{6}$. De acordo com a realidade das creches, é recorrente o fato de que as crianças não lavam as mãos após utilizar o banheiro e dependem da cuidadora para realizar higiene pessoal, favorecendo a contaminação oral - fecal e a disseminação de parasitoses intestinais ${ }^{7}$.

Segundo dados da Organização Mundial da Saúde (2018), 9.475 .765 crianças brasileiras necessitavam de tratamento preventivo para helmintíases transmitidas pelo solo ${ }^{8}$. Apesar das parasitoses intestinais não terem uma alta taxa de mortalidade, elas podem ser a causa de alguns sinais e sintomas 
importantes como: anemia, dor abdominal, diarreia, cólicas, náuseas, vômitos e febre ${ }^{9,10}$. Além disso, são capazes também de influenciar no desenvolvimento físico e cognitivo, inclusive causar reduções significativas no rendimento escolar ${ }^{11}$. No Brasil, nota-se uma redução de parasitose infantil, plausíveis decorrente da melhora sanitária e aspectos sociais ${ }^{12}$.

Dessa forma, o objetivo do trabalho é analisar os dados das principais pesquisas existentes no Brasil sobre enteroparasitoses entre pré-escolares e relacioná-las com aspectos sociais, econômicos e culturais.

\section{METODOLOGIA}

A pesquisa tem como tipo de estudo a revisão integrativa e surgiu a partir da pergunta norteadora, há diferença entre a prevalência de enteroparasitoses no século passado para a atualidade. Os operadores booleanos utilizados foram "prevalência e parasitose e crianças". Foi utilizado as bases de dados Lilacs (Literatura em Ciências da Saúde), Google Acadêmico e Scielo, Pubmed devido a relevância que essas bases de dados apresentam ao tema. A coleta de dados foi realizada em março de 2021. O recorte temporal foi de 30 anos com objetivo de comparar a prevalência de parasitos no século passado e avaliar se há diferença com o cenário atual.

Para a inclusão dos artigos, foram selecionados aqueles que foram realizados no Brasil, em português, de 1990 a 2020 e que apresentassem a prevalência de parasitos em crianças de 0 a 12 anos. Os critérios de exclusão foram pesquisas cuja faixa etária era acima de 12 anos, pesquisas estrangeiras, publicados antes de 1990, que não apresentassem o número da prevalência de parasitos ou fossem uma revisão sistemática. Para que facilitasse a busca pelos artigos elegíveis, foram utilizados filtros nas bases de dados. Nas bases Lilacs, Google Acadêmico e Scielo foi utilizado o filtro "1990-2020” enquanto que no PubMed foram utilizados os filtros "1990-2020", "portuguese”, "infant: 1-23 months", "preschool child: 25 years" e "child: 6-12 years"

Da amostra total encontrada nas bases de dados, a primeira etapa foi selecionar os artigos pela leitura do título e resumo. Aqueles que não atendessem a data, ao tema ou ao idioma foram descartados. Posteriormente, na segunda etapa, os artigos selecionados foram lidos na íntegra e aqueles que não cumpriram com os critérios de inclusão foram excluídos, restando apenas artigos com nível de evidência 3 ou 4. Esse processo foi realizado duas vezes, com pesquisadores diferentes, para diminuir erros ou vieses.

$\mathrm{Na}$ base de dados Lilacs, foram encontrados 56 artigos, desses 8 selecionados após primeira etapa e 5, após a segunda etapa. No Google Acadêmico foram encontrados 10.800 artigos destes,47 selecionados primeiramente e após a leitura completa, 25. No Scielo, a amostra inicial foi de 21 e após a primeira e segunda etapa restaram 05. No PubMed foram encontrados 295 artigos, destes 8 foram selecionados e após a segunda etapa apenas um artigo era elegível. A partir dessa amostragem, foram descartados 5 artigos que 
estavam repetidos nas bases de dados. Dessa maneira, a revisão sistemática contou com uma amostra final de 31 artigos e o fluxograma das etapas estão apresentadas abaixo (figura 1).

Figura 1 - Fluxograma das etapas da seleção dos artigos

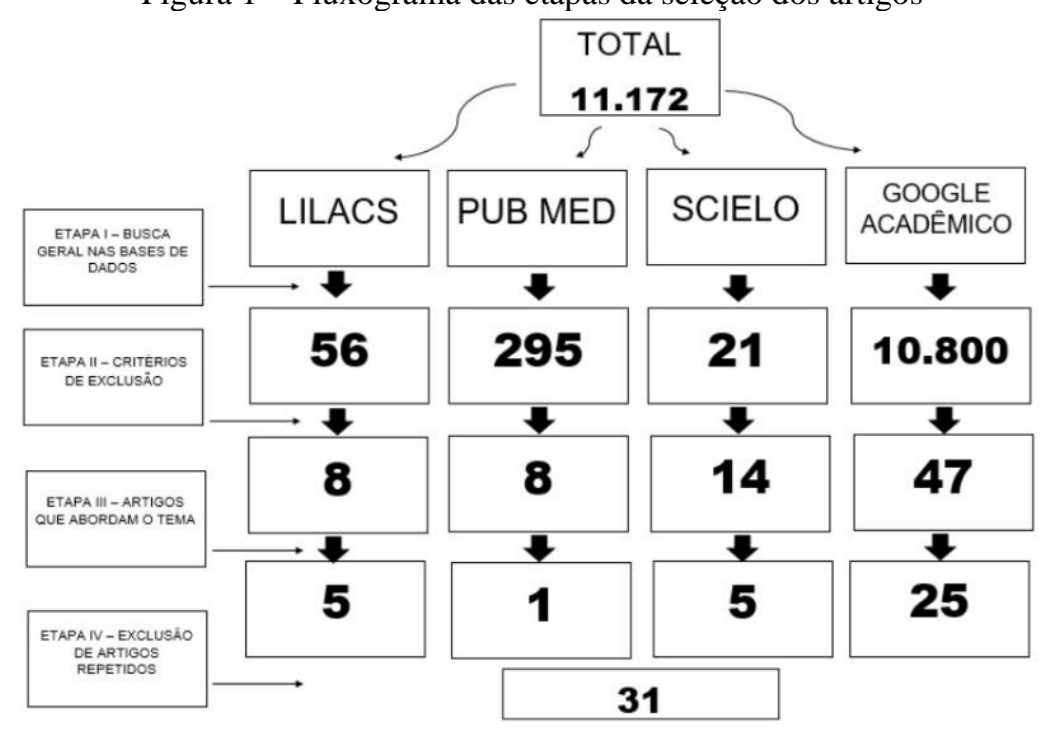

\section{RESULTADOS}

Foram encontradas 29 publicações a partir de 2001, contrastando com o número de 2 artigos encontrados entre 1990 e 2000 (tabela 1).

\begin{tabular}{|c|c|c|c|}
\hline \multicolumn{4}{|c|}{ Tabela 1- Relação entre a no e número de artigos catalogados } \\
\hline $1990-2000$ & $2001-2010$ & $2011-2020$ & Total \\
\hline 2 & 11 & 18 & 31 \\
\hline
\end{tabular}

Em relação as regiões do Brasil, os estudos foram feitos nas cinco regiões, prevalecendo o Sudeste com nove publicações (29\%), Sul com oito (26\%), Nordeste com sete publicações (22\%), seguido de Centro-Oeste com quatro publicações (13\%) e Norte com três (10\%), demonstrado na figura 2.

Figura 2 - Relação entre região do Brasil e número de artigos catalogados

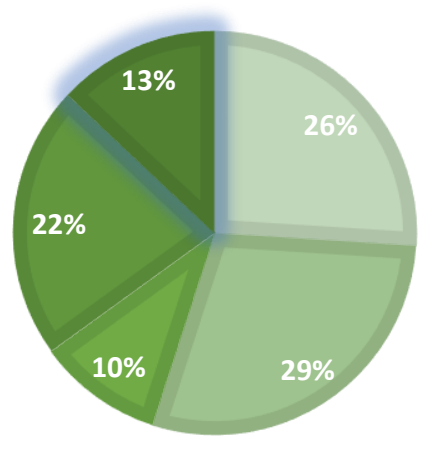

Sul Sudeste $\square$ Norte $\square$ Nordeste $\square$ Centro-Oeste 
Quando separados os artigos por região do Brasil, na região Sudeste a maior prevalência de parasitas está nos estudos de Espírito Santo e Rio de Janeiro. Na região Sul, prevalece em Santa Catarina e Rio Grande do Sul. No Centro-Oeste, predomina no Mato Grosso. No Nordeste, a Paraíba se sobressai. No Norte, não houve diferença muito significativa entre os estados de Tocantins, Pará e Amazonas. Também não houve discrepância expressiva entre as espécies de parasitas encontrados em cada região do Brasil.

Com relação ao método diagnóstico empregado, vinte trabalhos utilizaram um método parasitológico, oito publicações utilizaram dois métodos e outros três estudos utilizaram três métodos. Dentre os métodos usados, Hoffman, Pons e Janer foi o mais utilizado (56\%), seguido de Ritchie (9\%), Método Direto e Faust e cols. com 7\% cada. Baermann-Moraes e Blagg, cada, foram utilizados em 5\% das publicações. MIF, Kato-Katz, ELISA, Willis e Swab Anal estavam presentes em 2\% dos artigos (figura 3). Vale ressaltar que duas publicações usaram a análise de prontuários como forma de pesquisa de parasitas.

Figura 3 - Relação entre métodos diagnósticos e número de artigos

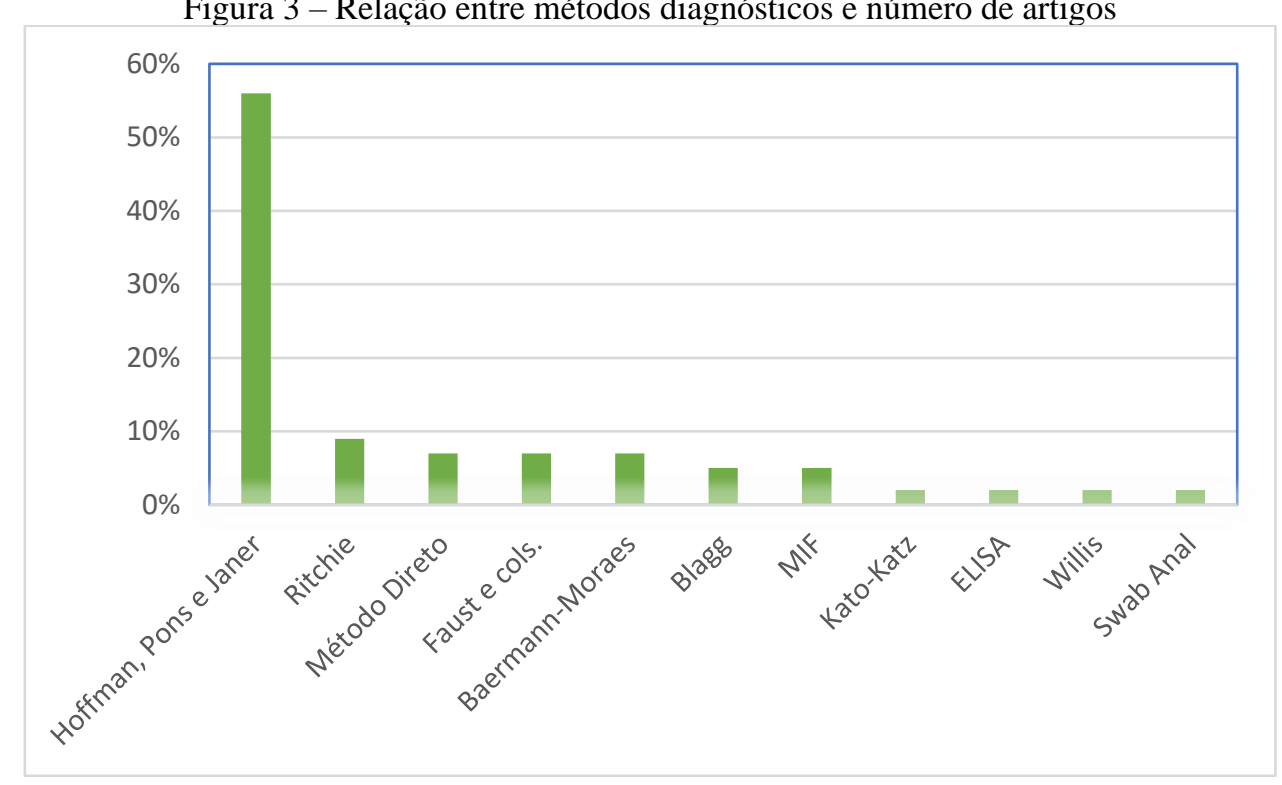

\section{DISCUSSÃO}

Quando comparamos a proporção de artigos em relação ao ano, nota-se maior prevalência de estudos a partir do ano de 2011, provavelmente devido a maior troca de informações com avanço da tecnologia na ciência. Com relação à origem dos estudos, a maioria é dos estados Sudeste e Sul, regiões com nível socioeconômico maior comparado às outras, o que demonstra que as outras regiões devem ser mais estudadas, principalmente Norte e Nordeste.

Os estudos dos anos 90 apresentam prevalência de parasitoses semelhantes aos comparados com os trabalhos realizados a partir do ano 2000, porém há uma pequena amostragem de artigos desse período o que dificulta a comparação. No trabalho de Moura et al, de uma amostra com 146 crianças de 6 a 11 anos, $30,8 \%$ se apresentaram positivas, enquanto que no estudo de Costa-Macedo et al, a positividade de parasitas 
foi de 54,5\% em uma amostra com 1381 crianças. Além da prevalência semelhante, os parasitas encontrados continuam os mesmos como Ascaris lumbricoides, Giardia lamblia, Ancilostomídeos $e$ Enterobius vermicularis ${ }^{13,14}$.

Também foi constatado a presença de pelo menos um parasita comensal em 21 artigos. O parasita Entamoeba coli se repetiu em 20 publicações, Endolimax nana em 15 e Iodamoeba butschlii em 5. Esse tipo de parasita não é capaz de produzir doença, mas pode servir como marcador de ausência ou precariedade de saneamento básico ${ }^{15}$. No estudo de Menezes et al, foi visto menor prevalência de parasitos nas creches as quais a população possuía melhores condições socioeconômicas e higiênico-sanitárias ${ }^{16}$. Vasconcelos et al, além de concluir que a prevalência foi maior nos moradores de casas com menor saneamento básico, filhos de mães com baixa escolaridade também apresentaram altos índices de infectados, demonstrando que os riscos da infecção é multifatorial ${ }^{17}$.

Na região sul, no Rio Grande Do Sul (RS) houve um aumento da prevalência de parasitose comparando o ano de 2012 no estudo de Costa CS et al que revelou parasitismo em 6,8\% das crianças e no estudo de 2013 de Incerti et al, no qual observou 67,2\% crianças parasitadas. Estes valores são discrepantes, mas Incerti et al (2013) evidenciou uma faixa etária maior de crianças, de 4 a 12 anos, enquanto o outro autor, crianças de 12 a 16 meses ${ }^{18,19}$. Ambos os estudos utilizaram o método de sedimentação espontânea, portanto, o aumento da prevalência não é justificado pela técnica empregada no exame de coproparasitológico. O estudo com menor prevalência de parasitoses foi realizado na capital do estado, Porto Alegre. Porém, o autor realizou sua pesquisa em um município pequeno do estado, Cacique Doble, em comparação com a população total, sendo justificável a maior prevalência na cidade menor ${ }^{16}$.

No estado de Goiás, a presença de parasita nas fezes de crianças foi decrescente de 2008 para 2019, Zaiden et al no seu estudo com 276 crianças de 0 a 6 anos, declarou uma porcentagem de $39,9^{20}$. Subsequente, em 2015, Silva et al revelou que 29,6\% de crianças no seu estudo estavam positivas para algum parasita, e por fim, em 2019 a presença de parasitas foi de 17,6\% ${ }^{21}$. Novamente os 3 estudos utilizaram o método HPJ. Dentre as cidades estudadas, a de maior prevalência é Rio Verde, de maior população, seguido de Itapurunga e Ceres que são cidades menores, no interior do estado, justificando uma menor quantidade de resultados positivos.

O estado de São Paulo, mostrou um aumento na prevalência de parasitose. Em 1997, Moura et al afirmou no seu estudo que 24,6\% de crianças estavam parasitadas, já Biscegli et al, evidenciou 29,2\%. Estes dados vão em contrapartida da realidade atual, pois mesmo sendo em cidades diferentes, hoje em dia se tem um maior avanço da medicina e dos meios de transmissão, possibilitando uma menor prevalência de parasitos $^{13,22}$.

Com relação ao método diagnóstico empregado, desconsiderando o método Hoffman, Pons e Janer que foi amplamente utilizado, houve bastante variação de métodos entre os artigos. A maioria dos 
pesquisadores utilizaram o método de Hoffman, Pons \& Janer, que foi descrito por Lutz. Este método se baseia na técnica de sedimentação espontânea, um método de fácil execução e baixo custo, que não exige equipamentos especiais e permite o diagnóstico de um ou mais parasitos, além de possuir uma boa sensibilidade $^{23}$. O método Kato-Katz foi utilizado em apenas $2 \%$ das publicações. É uma técnica quantitativa, também de baixo custo, que permite o armazenamento e transporte de lâminas em temperatura ambiente por meses, sem prejudicar os resultados ${ }^{23}$. No entanto, vale ressaltar que esses métodos são inespecíficos.

\section{CONSIDERAÇÕES FINAIS}

Após a realização desse estudo, conclui-se que devido às diversas correlações entre enteroparasitoses e perfis socioeconômico-culturais, a melhor alternativa para a redução desta prevalência seria optar pela educação sanitária desde a infância, tendo em conta que o maior foco de transmissão são as crianças, por apresentarem hábitos de higiene não convencionais. Além das crianças, a orientação aos pais também é fundamental uma vez que baixos níveis de escolaridade estão relacionados a alta prevalência de parasitoses. É necessário a continuidade nesta temática para futuramente ser possível realizar outras comparações, principalmente entre os estados do Brasil. Tendo como base este estudo, cabe-se a realização de mais levantamentos de enteroparasitoses infantil em regiões com menor taxa de saneamento básico e nos estados Norte e Centro-Oeste que possuem menos publicações a respeito do tema. 


\section{REFERÊNCIAS}

1. Silva Jd. Parasitismo intestinal em pré-escolares no município de Teresina, Piauí:estudo transversal em creches públicas no período de novembro de 2017 a junho de 2018.

Fundação Oswaldo Cruz [internet]. Teresina 2018 [citado 2020 Mar. 04]. Disponível em:

https://www.arca.fiocruz.br/handle/icict/34580.

2. Sistema Nacional de Informações sobre Saneamento. Diagnóstico dos Serviços de Água e Esgotos - 2018. Ministério do Desenvolvimento Regional [internet]. Brasília 2019

[citado em 2020 Mar. 05]. Disponível em:

http://www.snis.gov.br/diagnostico-anual-agua-e-esgotos/diagnostico-dos-servicos-de-aguae-esgotos-2018.

3. Souza CZd, Zetermann CD. Parasitoses intestinais em crianças de centros de educação infantil (CEIS) do município de Barretos, estado de São Paulo, Brasil. IFSP.

[internet]. Barretos 2013 [citado em 15 Mar 2020]. Disponível em:

http://docplayer.com.br/62306739-Parasitoses-intestinais-em-criancas-de-centros-de-educa

cao-infantil-ceis-do-municipio-de-barretos-estado-de-sao-paulo-brasil.html

4. Barbosa ALNH, Costa JSM. Oferta de creche e participação das mulheres no mercado de trabalho no brasil. Instituto de Pesquisa Econômica Aplicada [internet]. Brasília 2017 [citado 2020 Mar. 04]. Disponível em: mhttp://repositorio.ipea.gov.br/bitstream/11058/7805/1/bmt_62_oferta.pdf.

5. Santos Jd, Duarte ARM, Gadotti G, Lima LM. Parasitoses intestinais em crianças de creche comunitária em Florianópolis, SC, BRASIL. Rev Patol Trop Vol [internet]. Florianópolis 2014 [citado 2020 Mar. 04]. Disponível em: http://docplayer.com.br/19208193-Parasitoses-intestinais-em-criancas-de-creche-comunitaria a-em-florianopolis-sc-brasil.html.

6. Silva TCT et al. Cuidado Integral à saúde da criança no contexto das políticas públicas. Saúde Coletiva [internet] Barueri 2019 [citado em 2021 Out. 31]. Disponível em: http://revistas.mpmcomunicacao.com.br/index.php/saudecoletiva/issue/view/26/25.

7. Uchoa CMA et al. Enteroparasitoses em Crianças de Creche [internet]. [Acesso em:

13 mar. 2020]. Disponível em: https://www.ufmg.br/congrext/Saude/Saude80.pdf.

8. WHO. Soil-transmitted helminthiases: Number of children (Pre-SAC and SAC)

requiring preventive chemotherapy for soil-transmitted helminthiases 2018. [internet]. Brazil

2018 [cited in15 Mar 2020]. Available in: http://apps.who.int/neglected_diseases/ntddata/sth/sth.html.

9. Secretaria de Estado da Saúde do Rio de Janeiro. Parasitoses Intestinais. BVS [internet]. Rio de Janeiro 2005 [citado em 2020 Mar. 05]. Disponível em: http://bvsms.saude.gov.br/bvs/dicas/74parasitoses.html.

10. Belo VS et al. Fatores associados à ocorrência de parasitoses intestinais em uma população de crianças e adolescentes. Rev. paul. pediatr. [internet]. São Paulo 2012 [citado

em 2020 Mar. 05]. Disponível em:

http://www.scielo.br/scielo.php?script=sci_arttext\&pid=S0103-05822012000200007. 
11. Clerici DJ, Pigatto AGS. Associação entre parasitoses intestinais e rendimento escolar: revisão sistemática [internet]. 2015. [Acesso em: 05 mar. 2020]. Disponível em: https://periodicos.ufn.edu.br/index.php/disciplinarumS/article/view/1101.

12. Saúde da criança. Manual de acompanhamento da criança v.3-Parasitoses Intestinais mais frequentes na criança. [Internet]@ 2016 - 2018 [citado 2021 Mar 30]. Disponível em: http://saudeemacao.saude.sp.gov.br/crianca-3/parasitoses-intestinais-mais-frequentes-nacrianca/\#: :text=As\%20parasitoses\%20intestinais\%20s\%C3\%A3o\%20um,organiza\%C3\%A7\%C3\%A3o\%20 dos\%20servi\%C3\%A7os\%20de\%20sa\%C3\%BAde.

13. Moura EC et al. Prevalência de parasitose intestinal em escolares da primeira serie de uma escola publica [internet]. [Acesso em: 29 mar. 2021]. Disponível em: https://pesquisa.bvsalud.org/portal/resource/pt/lil211803.

14. Costa-Macedo Ld et al. Parasitoses intestinais em pré-escolares de favelas da cidade do Rio de Janeiro, Brasil; [internet]. [Acesso em: 29 mar. 2021]. Disponível em: https://pubmed.ncbi.nlm.nih.gov/9878918/.

15. Cavalcante UMB, Melo SAL, Lima CMBL. Enteroparasitoses na População Infantil sua Prevalência e Modelos de Decisão Utilizados: Revisão Sistemática. [internet] [Acesso em: 30 mar. 2021]. Disponível em: https://periodicos.unicesumar.edu.br/index.php/saudpesq/article/view/4497.

16. Menezes AL et al. Prevalência de enteroparasitoses em crianças de creches públicas da cidade de Belo Horizonte, Minas Gerais, Brasil [internet]. [Acesso em: 29 mar. 2021]. Disponível em: https://pesquisa.bvsalud.org/portal/resource/pt/lil-476766.

17. Vasconcelos IAB et al. Prevalência de parasitoses intestinais entre crianças de 4-12 anos no Crato, Estado do Ceará: um problema recorrente de saúde pública [internet]. [Acesso em: 30 mar. 2021]. Disponível em: https://periodicos.uem.br/ojs/index.php/ActaSciHealthSci/article/view/8539/8539.

18. Costa CdS et al. Prevalência de parasitoses em crianças de 12 a 16 meses atendidas em unidades de saúde de Porto Alegre, Rio Grande do Sul [internet]. [Acesso em: 29 mar. 2021]. Disponível em: https://pesquisa.bvsalud.org/portal/resource/pt/lil-691093.

19. Incerti $\mathbf{J}$ et al. Prevalência de parasitoses intestinais entre crianças da comunidade indígena de Cacique Doble/RS [internet]. [Acesso em: 29 mar. 2021]. Disponível em: https://www.lume.ufrgs.br/handle/10183/78460

20. Zaiden MF et al. Epidemiologia das parasitoses Intestinais em crianças de creches de Rio Verde-GO [internet]. [Acesso em: 29 mar. 2021]. Disponível em: https://www.revistas.usp.br/rmrp/article/view/265.

21. Silva AO et al. Epidemiologia e prevenção de parasitoses intestinais em crianças das creches municipais de Itapuranga - GO [internet], [Acesso em: 30 mar. 2021]. Disponível em: http://www.revista.fmb.edu.br/index.php/fmb/article/viewFile/18/14.

22. Biscegli TS et al. Estado nutricional e prevalência de enteroparasitoses em crianças matriculadas em creche [internet]. [Acesso em: 29 mar. 2021]. Disponível em: https://www.scielo.br/scielo.php?pid=S010305822009000300009\&script=sci_arttext.

23. A Rabello et al. Diagnóstico Parasitológico, Imunológico e Molecular da Esquistossomose Mansoni [internet]. Rio de Janeiro: Fiocruz; 2008. [Acesso em: 29 fev. 2021]. Disponível em: http://books.scielo.org/id/37vvw/pdf/carvalho-9788575413708-33.pdf. 